\title{
Primeiro registro de Pseustes sulphureus (Wagler, 1824) (Serpentes: Colubridae) no Estado de Alagoas, Nordeste do Brasil
}

\author{
Barnagleison Silva Lisboa ${ }^{1 *}$ \\ Ingrid Carolline Soares Tiburcio ${ }^{2}$ \\ Selma Torquato da Silva ${ }^{1}$ \\ Gabriel Omar Skuk Sugliano ${ }^{1,3}$ \\ ${ }^{1}$ Setor de Zoologia, Museu de História Natural, Universidade Federal de Alagoas \\ Avenida Aristeu de Andrade, 452, CEP 57051-090, Maceió - AL, Brasil \\ ${ }^{2}$ Departamento de Zoologia, Instituto de Biociências, Universidade Estadual Paulista \\ Caixa Postal 199, CEP 13506-900, Rio Claro - SP, Brasil \\ ${ }^{3}$ Setor de Biodiversidade e Ecologia, Instituto de Ciências Biológicas e da Saúde \\ Universidade Federal de Alagoas, Praça Afrânio Jorge, $\mathrm{s} / \mathrm{n}^{\circ}$, \\ Prado, CEP 57010-020, Maceió - AL, Brasil. \\ *Autor para correspondência \\ bslgleison@gmail.com
}

\section{Resumo}

O gênero Neotropical Pseustes Fitzinger, 1843 possui ampla distribuição nas florestas úmidas dos países das Américas Central e do Sul. No Brasil, P. sulphureus ocorre nos biomas Floresta Amazônica e Mata Atlântica. Em novembro de 2007 foi obtido o primeiro registro de $P$. sulphureus para o Estado de Alagoas, no município de Campo Alegre, Nordeste do Brasil. O espécime de P. sulphureus está depositado na coleção herpetológica do Museu de História Natural, Universidade Federal de Alagoas (MUFAL).

Unitermos: Alagoas, Campo Alegre, primeiro registro, Pseustes sulphureus

\section{Abstract}

First record of Pseustes sulphureus (Wagler, 1824) (Serpentes: Colubridae) in the state of Alagoas, northeastern Brazil. The Neotropical snake genus Pseustes Fitzinger, 1843 has a broad distribution in the humid forests of Central and South America. In Brazil, Pseustes sulphureus occurs in the biomes of the Amazonian and Atlantic forests. In November 2007, the first record of this species was obtained for the state of Alagoas, in the municipality of Campo Alegre, northeastern Brazil. The specimen of $P$. sulphureus is deposited in the herpetological collection of the Museu de História Natural, Universidade Federal de Alagoas (MUFAL).

Key words: Pseustes sulphureus, first record, Campo Alegre, Alagoas 
A serpente Neotropical Pseustes sulphureus (Wagler, 1824) possui ampla distribuição nas florestas úmidas dos países da América do Sul, ocorrendo no Peru, Equador, Brasil, Guianas e Trinidad (Peters e Orejas-Miranda, 1970). No Brasil, P. sulphureus ocorre na Floresta Amazônica (Cunha et al., 1985; Frota et al., 2005) e na Mata Atlântica, com registros nesta última para os Estados de São Paulo, Rio de Janeiro, Bahia, Paraíba e Ceará (Marques e Calleffo, 1997; Franco et al., 1998; Argôlo, 2004; Rocha, et al., 2004; Borges-Nojosa et al., 2006; Santana et al., 2008).

No dia 01 de novembro de 2007, foi coletado um espécime adulto de P. sulphureus (Figura 1) na Fazenda Pindoba, fragmento de Mata Atlântica, localizado no município de Campo Alegre (0945'32"S e 036 $14^{\circ}$ '9'W, $121 \mathrm{~m}$ de altitude), no leste do Estado de Alagoas, sendo este o primeiro registro para o Estado (Figura 2). O espécime foi identificado, apresentando as seguintes características de diagnose da espécie: 215 escamas ventrais; 122 pares de escamas subcaudais; escamas dorsais quilhadas e com um par de fossetas apicais; placa anal única. Internasais subquadrangulares em contato com rostral, nasais anteriores, posteriores e pré-frontais. Pré-frontais subquadrangulares em contato com rostral, nasal posterior, loreal, pré-ocular e supra-ocular; frontal subtriangular contactando-se com pré-frontais, supra-ocular e parietais; 8 supra-labiais; 10 infra-labiais; 1 pré-ocular; 3 pós-oculares; 1 supra-ocular. Pós-mentais anteriores unidas na linha mediana. Pós mentais posteriores separadas medianamente por pequenas escamas gulares imbricadas. 2,20m de comprimento total; $0,47 \mathrm{~m}$ de comprimento da cauda. Teve sua identificação confirmada por Adriano L. Silveira (MN-UFRJ) e encontrase depositado na coleção herpetológica do Museu de História Natural - Universidade Federal de Alagoas, sob o registro MUFAL 7124.

O local onde foi encontrada a serpente é um remanescente de Mata Atlântica em área de tabuleiro, com cerca de 790ha, $130 \mathrm{~m}$ de altitude média e árvores ultrapassando os $30 \mathrm{~m}$ de altura. Inclui-se na região da faixa bioclimática entre 0 e 90 dias biologicamente secos ao longo de cada ano, o que o caracteriza como floresta ombrófila. Litologicamente a área é formada por arenitos terciários do Grupo de Barreiras, sob forma de tabuleiros aplainados com diferentes tipos de solo (Latossolo Vermelho Amarelo distrófico em associação com Podzólico Vermelho Amarelo latossólico e Podzólico Vermelho Amarelo) e forte indicativo de poucas alterações na sua cobertura vegetal causadas por interferências paleoclimáticas, a partir da sua última mudança (Assis, 2000).

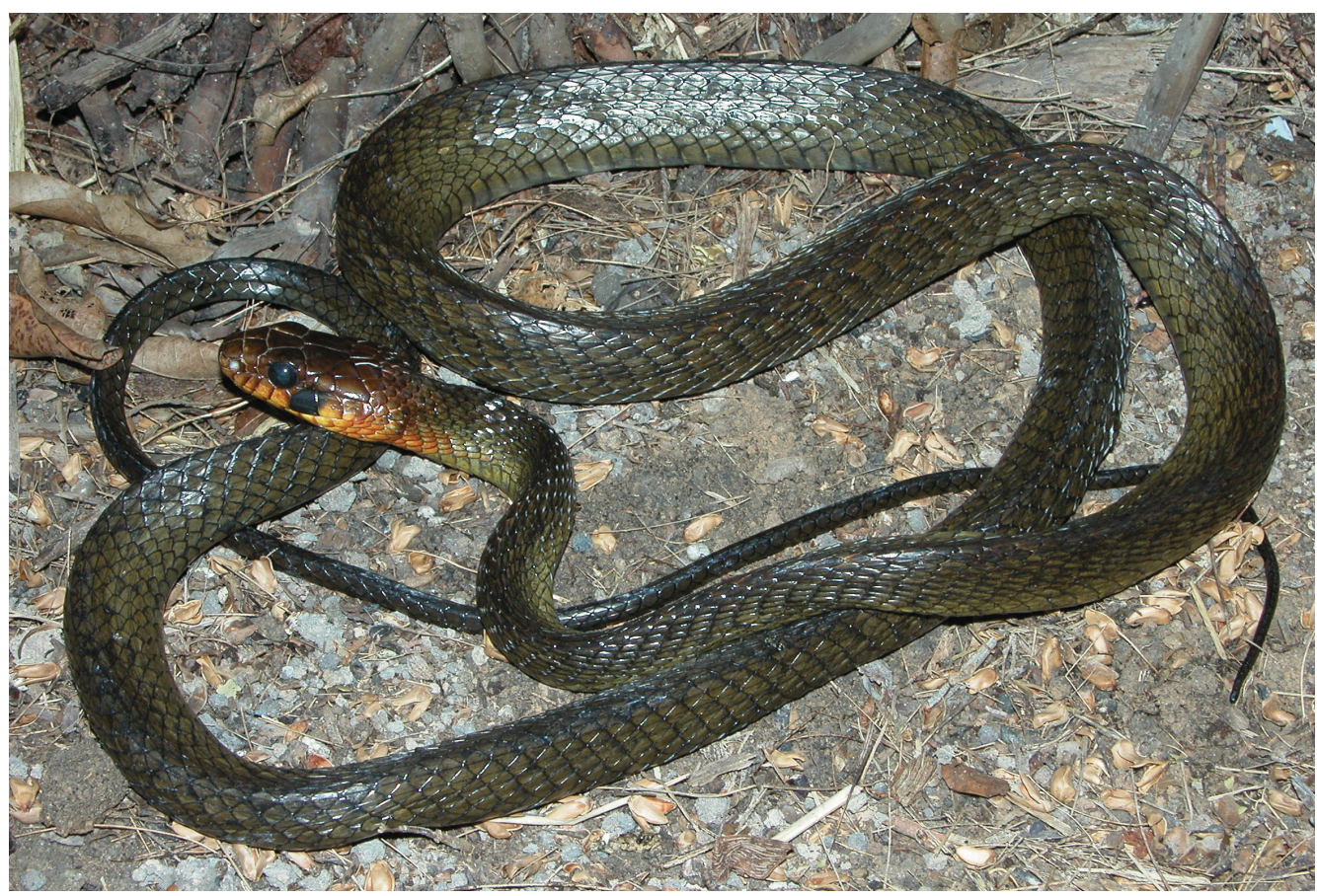

FIGURA 1: Espécime de Pseustes sulphureus coletado (MUFAL 7124) na Fazenda Pindoba, fragmento de Mata Atlântica no município de Campo Alegre, Alagoas. (Foto: Gabriel Skuk). 


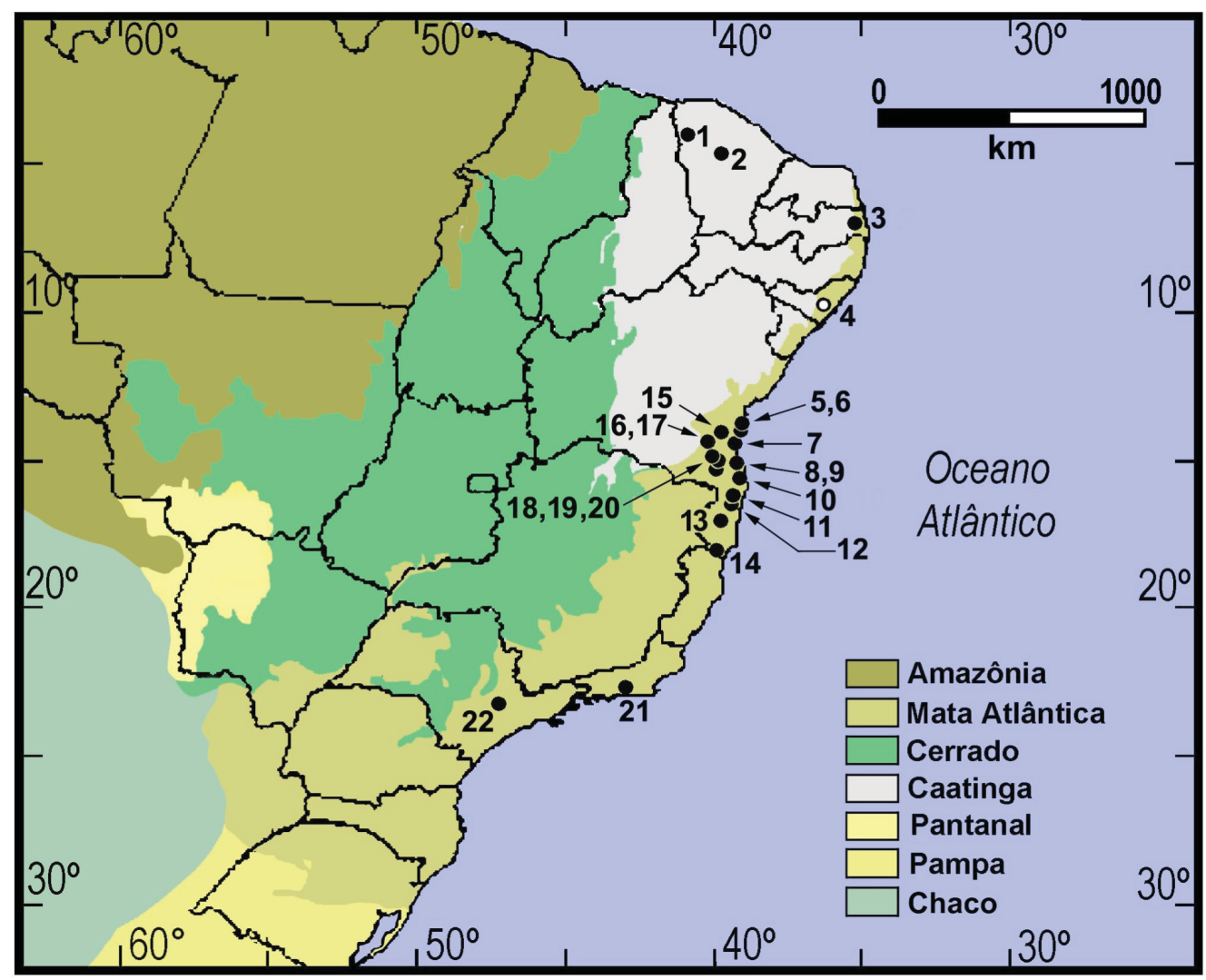

FIGURA 2: Registros de Pseustes sulphureus em remanescentes de Mata Atlântica. A nova ocorrência citada neste estudo está indicada pelo círculo aberto. Localidades: (1) Parque Nacional de Ubajara, município de Ubajara, CE; (2) Maciço do Baturité, município de Pacoti, CE; (3) município de João Pessoa, PB; (4) Fazenda Pindoba, município de Campo Alegre, AL; (5) município de Ituberá, BA; (6) município de Camamú, BA; (7) município de Itacaré, BA; (8) município de Ilhéus, BA; (9) município de Buerarema, BA; (10) município de Canavieiras, BA; (11) município de Porto Seguro, BA; (12) município de Itabela, BA; (13) município de Itamarajú, BA; (14) município de Mucuri, BA; (15) município de Ibirapitanga, BA; (16) município de Itagibá, BA; (17) município de Itagí, BA; (18) município de Almadina, BA; (19) município de Ibicaraí, BA; (20) município de Jussari, BA; (21) município de Maricá, RJ e (22) município de Paranapiacaba, SP.

Tiburcio et al. (2008) realizaram neste mesmo remanescente de floresta outro estudo herpetofaunístico, registrando o anuro Macrogenioglottus alipioi Carvalho, 1946 (família Cycloramphidae), considerado raro, mesmo com sua distribuição geográfica conhecida desde o sul do Estado da Bahia, de onde é descrita, até o Estado de São Paulo. Esses dois novos registros evidenciam a relevância da conservação do remanescente referido, já recomendado por Assis (2000), como uma das áreas a ser usada nas pesquisas de reintrodução de espécies da fauna consideradas extintas no Estado de Alagoas.

\section{Agradecimentos}

A Lahert L. Araújo e Phoeve Macario por ajuda em campo. AAdriano L. Silveira, doutorando do Museu
Nacional (UFRJ) por confirmação da espécie. A Usina Porto Rico por nos conceder permissão e apoio logístico na área estudada. À FAPESP por auxílio financeiro. Aos consultores anônimos por suas sugestões importantes que contribuíram para a melhoria do manuscrito.

\section{Referências}

Argôlo, A. J. S. 2004. As serpentes dos cacauais do sudeste da Bahia. Ed. Editus, Ilhéus, Brasil, 260pp.

Assis, J. S. 2000. Biogeografia e conservação da biodiversidade - Projeções para Alagoas. Ed. Catavento, Maceió, Brasil, 200pp. Borges-Nojosa, D. M.; Loebmann, D.; Lima, D. C.; Melo, J. C. L.; Mai, A. C. G. 2006. Reptilia, Colubridae, Pseustes sulphureus: Distribution extension, new state record. Check List, 2 (3): 79-81. Cunha, O. R.; Nascimento, F. P.; Ávila-Pires, T. C. S. 1985. Os répteis da área de Carajás, Pará, Brasil (Testudines e Squamata). 
Publicações Avulsas do Museu Paraense Emilio Goeldi, 40: 9-92.

Franco, F. L.; Sugliano, G. O. S.; Porto, M.; Marques, O. A. V. 1998. Répteis da Estação Vera Cruz (Porto Seguro, Bahia). Publicação Técnico-Científica Estação Vera Cruz, 3: 1-39.

Frota, J. G.; Santos-Jr, A. P.; Chalkidis, H. M.; Guedes, A. G. 2005. As serpentes da região do baixo rio Amazonas, oeste do estado do Pará, Brasil (Squamata). Biociências, 13 (2): 211-220.

Marques, O. A. V.; Calleffo, M. E. 1997. Geographic distribution: Pseustes sulphureus. Herpetological Review, 28 (3): 160.

Peters, J. A.; Orejas-Miranda, B. 1970. Catalogue of the Neotropical Squamata, part 1, Snakes. Bulletin of the United States National Museum, 297: 1-347.
Rocha, C. F. D.; Bergallo, H. G., Pombal Jr, J.; Geise, L.; Van Sluys, M.; Fernandes, R.; Caramaschi, U. 2004. Fauna de anfíbios, répteis, mamíferos do estado do Rio de Janeiro, sudeste do Brasil. Publicações Avulsas do Museu Nacional do Rio de Janeiro, 104: 3-23.

Santana, G. G.; Vieira, W. L. S.; Pereira-Filho, G. A.; Delfim, F. R.; Lima, Y. C. C.; Vieira, K. S. 2008. Herpetofauna em um fragmento de Floresta Atlântica no Estado da Paraíba, região nordeste do Brasil. Biotemas, 21 (1): 75-84.

Tiburcio, I. C. S.; Lisboa, B. S.; Haddad, C. F. B. 2008. Amphibia, Anura, Cycloramphidae, Macrogenioglottus alipioi: Distribution extension, state of São Paulo and Alagoas, Brazil. Check List, 4 (4): $455-457$. 